\title{
SCN1A, SCN1B, and GABRG2 gene mutation analysis in Chinese families with generalized epilepsy with febrile seizures plus
}

\author{
Huihui Sun · Yuehua Zhang · Jianmin Liang · \\ Xiaoyan Liu $\cdot$ Xiuwei Ma $\cdot$ Husheng Wu \\ Keming Xu $\cdot$ Jiong Qin $\cdot$ Yu Qi $\cdot$ Xiru Wu
}

Received: 10 March 2008/Accepted: 21 May 2008/Published online: 20 June 2008

(C) The Japan Society of Human Genetics and Springer 2008

\begin{abstract}
Generalized epilepsy with febrile seizures plus (GEFS+; MIM\#604233) is a familial epilepsy syndrome characterized by phenotypic and genetic heterogeneity. It was associated with mutations in the neuronal voltagegated sodium channel subunit gene (SCN1A, SCN2A, $S C N 1 B$ ) and ligand-gated gamma aminobutyric acid receptors genes $(G A B R G 2, G A B R D)$. We investigated the roles of $S C N 1 A, S C N 1 B$, and GABRG2 mutations in the etiology of Chinese GEFS+ families. Genomic deoxyribonucleic acid (DNA) was extracted from peripheral blood lymphocytes of 23 probands and their family members. The sequences of $S C N 1 A, S C N 1 B$, and GABRG2 genes were analyzed by polymerase chain reaction (PCR) and direct sequencing. The major phenotypes of affected members in the 23 GEFS + families exhibited FS and FS+, whereas rare phenotypes afebrile generalized tonic-clonic seizures (AGTCS), myoclonic-astatic epilepsy (MAE), and partial seizures were also observed. A novel SCN1A mutation, p.N935H, was identified in one family and
\end{abstract}

H. Sun · Y. Zhang $(\varangle) \cdot$ X. Liu $\cdot$ X. Ma · J. Qin ·

Y. Qi · X. Wu

Peking University, First Hospital, No. 1 Xian Men Street,

Xicheng District, Beijing 100034, People's Republic of China

e-mail: zhangyhd@bjmu.edu.cn

J. Liang

Jilin University, First Hospital, Changchun 130021, China

H. $\mathrm{Wu}$

Beijing Children's Hospital, Beijing 100045,

People's Republic of China

K. Xu

Capital Institute of Pediatrics, Beijing 100020,

People's Republic of China another novel mutation in GABRG2, p.W390X, in another family. However, no $S C N I B$ mutation was identified. The combined frequency of $S C N 1 A, S C N 1 B$, and GABRG2 mutations was $8.7 \%(2 / 23)$, extending the distribution of SCN1A and GABRG2 mutations to Chinese GEFS+ families. There were still unidentified genes contributing to the pathogenesis of GEFS+.

Keywords Generalized epilepsy with febrile seizures plus (GEFS+) - SCN1A $\cdot S C N 1 B \cdot G A B R G 2 \cdot$ Mutation · Chinese

\section{Introduction}

Generalized epilepsy with febrile seizures plus (GEFS+; MIM\#604233) is a familial epilepsy syndrome characterized by phenotypic heterogeneity including febrile seizures (FS), febrile seizures plus (FS+), FS/FS+ and generalized or partial seizures, myoclonic-astatic epilepsy (MAE), and severe myoclonic epilepsies of infancy (SMEI) (Scheffer and Berkovic 1997; Singh et al. 2001). GEFS+ is genetically heterogeneous and constitutes the family of "channelopathies." Mutations in genes encoding various subunits of neuronal voltage-gated sodium channels SCN1A, SCN1B, and SCN2A and subunits of gamma aminobutyric acid $(\mathrm{GABA})_{\mathrm{A}}$ receptors $G A B R G 2$ and $G A B R D$ were identified to be associated with GEFS+ (Escayg et al. 2000; Wallace et al. 1998; Baulac et al. 2001; Sugawara et al. 2001a; Dibbens et al. 2004). However, mutations in $S C N 2 A$ were only detected in one Japanese GEFS+ family and the GABRD gene in an Australian family. By contrast, SCN1A, SCNIB, and GABRG2 genes may be more frequently involved in the etiology of GEFS+ (Baulac et al. 2001; Mulley et al. 2005; Scheffer et al. 
2007). To further clarify the pathogenic roles of SCN1A, $S C N 1 B$, and $G A B R G 2$ genes, we performed mutation analysis in 23 Chinese GEFS+ families.

\section{Materials and methods}

Epileptic classification and patient ascertainment

The phenotypes of GEFS + included FS, FS+, FS/FS+ and generalized or partial seizures, MAE, and SMEI. FS+ is used to describe individuals with FS extending beyond the age of 6 years or with afebrile generalized tonic-clonic seizures (AGTCS) (Scheffer and Berkovic 1997). The detailed inclusion criteria of GEFS + were families where two or more individuals had a GEFS + phenotype, of which at least one had FS+.

This study recruited 23 unrelated Chinese GEFS+ families. They were examined at Peking University First Hospital from February 2005 to July 2007. Written informed consent was obtained from all participants. Consanguinity was not present in any of these families. The diagnosis of phenotypes was according to ILAE (Commission on Classification and Terminology of the International League Against Epilepsy 1989). The phenotypes of the 23 probands were: two FS alone, ten FS+, one FS and partial seizures, five FS + and partial seizures, one FS+ and myoclonic seizures, two MAE, one partial epilepsy alone, and one benign childhood epilepsy with centrotemporal spikes (BECT). Detailed clinical phenotypes were performed on all available family members, and seizure types and epileptic syndromes were classified. There were 127 affected members in 23 families, ranging from two to 22 affected members in each family. The affected members exhibited typical FS alone 48.8\% (62/ 127), FS + 31.5\% (40/127), FS/FS+ and partial seizures 4.7\% (6/127), AGTCS 7.9\% (AGTCS, 10/127), and MAE $1.6 \%$ (2/127). The following phenotypes occurred in seven members: one partial seizure alone, one FS+ and myoclonic seizures, one BECT, three unclassified epilepsy, and one unconfirmed epilepsy.

\section{Mutation analysis}

We initially tested 23 probands for $S C N 1 A, S C N 1 B$, and GABRG2 mutations. The corresponding segments of their family members of the probands with mutations were then examined for cosegregation analysis. Samples with alterations were sequenced in both directions from two independent polymerase chain reaction (PCR) products. To confirm that these alterations were mutations and not polymorphisms, genomic deoxyribonucleic acid (DNA) from 50 healthy Chinese controls of Han ethnicity was analyzed. None of the controls had a familial or personal history of febrile seizures or epilepsy.

Genomic DNA was extracted from peripheral blood lymphocytes by a simple salting out procedure (Miller et al. 1988). The coding region and flanking intronic regions of $S C N 1 A, S C N 1 B$, and GABRG2 were screened for mutations using PCR and direct sequencing. PCR was performed using primers as in previous studies (Wallace et al. 1998; Ohmori et al. 2002; Harkin et al. 2002).

\section{Results}

SCN1A, SCN1B, and GABRG2 mutation analysis

We recruited 23 GEFS + families with autosomal dominant inheritance in which the pedigree showed that two or more consecutive generations had affected members. Bilineal inheritance was present in three of the 23 GEFS + families involving three affected members. Of the 23 probands examined, one novel SCN1A mutation and another novel $G A B R G 2$ mutation were identified in two GEFS + families, respectively. Then we further examined the family members of the two families to track the coinheritance of phenotype with genotype. No SCNIB mutation was identified in any of these families.

The SCN1A mutation c.2803A > C/p.N935H

In family 1 (Fig. 1a), the proband (III-1) is a 5-year-old boy. He developed the first attack of FS when he was 1 year and 7 months, and the seizure type was generalized tonic-clonic (GTCS), which persisted for seconds. At age 2 years and 4 months, the second febrile seizures occurred. A week later, he had one AGTCS. Subsequently, he had a hemiclonic seizure for several minutes without fever. At age 3 years and 3 months, he was referred to our hospital, and video electroencephalogram (EEG) examination was conducted. The results showed multiple generalized slow waves with some spike discharges while awake and bilateral frontal spikes and spike waves during sleep. When he was 3 years and 8 months, partial seizures preceded by fever occurred. He was diagnosed with FS+ and partial seizures and was administered phenobarbital. Since then, he was seizure free to our study. There were four affected members in family 1 . The father (II-3), aunt (II-1) and uncle (II-2) of the proband each had FS once at age 34 years; their seizure type was GTCS. His mother had no personal or familial history of febrile seizures or epilepsy. To date, we have not been able to obtain blood samples of other family members, so we cannot perform further cosegregation analysis. 
A base substitution c.2803A $>C$ in exon 15 was detected in the proband and his father, and it was not found in his mother. The c.2803A $>\mathrm{C}$ transversion resulted in the substitution of neutral asparagine for a positively charged histidine p.N935H (Fig. 1b). This substitution was ascertained by reamplification and sequencing in the reverse primers. The entire coding region of SCNIA was sequenced, and no other alteration was found. It was not present in the 50 Chinese controls. The pathogenic function of the mutation was supported by the fact that it was located in the pore regions DIIS5-S6 linkers of the sodium channel $\alpha-1$ subunit. The conservative analysis of SCN1A

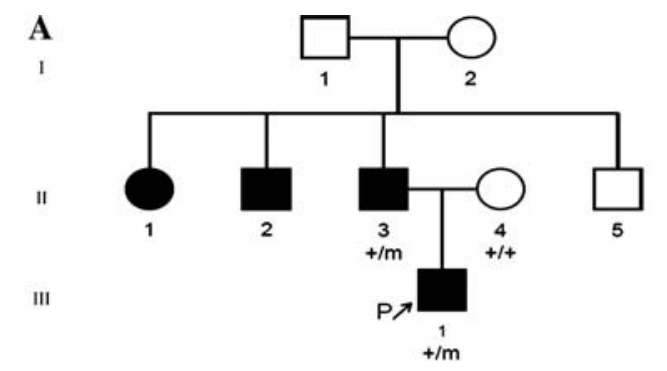

B

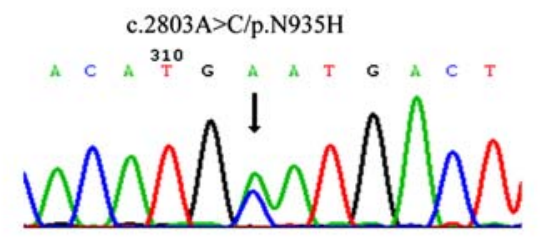

\section{C}

SCN1A_Human SCN1A_Rat SCN2A_Human SCN3A_Human SCN4A_Human SCN5A_Human SCN8A_Human SCN9A_Human SCN11A_Human SCN12A_Human Takifugu Danio rerio Electric eel Drosophila

\begin{abstract}
p.N935H
I

SYKDCYCKIASDCQUPRMTMNDFFHSFLIVFRVLCCEHIETMWDCMEVA-GOAMCLTVF 972 SYKDCVCKIATDCKLPRTHM DFFHSFLIVFRVLCGEWIETMMDCMEVA-GOMMCLTVF 972 SYKECVCKISNDCELPRTHM DFFHSFLIVFRVLCGEWIETMHDCMEVA-GQTMCL.TVF 963 SYKECVCKINDDCTLPRWHM DFFHSFLIVFRVLCCEWIETMMDCMEVA--GQTMCLIVFF 964 SYKECVCKIALDCNLPRTHM DFFHSFLIVFRILCGEWIETMMDCMEVA--GQAMCL.TVF 782 NYSELRD-SDSGLLPRTMMDFFHAFLIIFRILCCEWIETMMDCMEVS-GOSLCLLVF 919 SYKECYCKINQDCELPRTHM DFFHSFLIVFRULCGEWIETMMDCMEVA-GOAMCLIVF 957 SYKECVCKINDDCTLPRTHM DFFHSFLIVFRVLCGEWIETMHDCMEVA-GOAMCLIVY 948 SPKLCCNPTGPTVSCLRHMHMGDFWHSFLVVFRILOGEWIENMEECMOEANASSSICCVIVF 791 SPKLCNPTGPTVSCLRHMMGDFWHSFLVVFRILOGEWIEMMECMOEANASSSI.CVIVF 791 NYKDCYCKIARDCELPRWHM DFFHSFLIVFRVLCGEWIETMWDCMEVA--GOMMCLIVF 722 SYGECVCKISSDCMLPRMHM DFFHSFLIVFRVLCGEWIETMMDCMEVA-GQPLCL.TVF 937 NYKEYYCKISDDCELPRTHM DFFHSFLIVFRALOGEWIETMMDCMEVG--GVPWCLAVY 770 NYHDHKDRFPDG-DLPRWNFTDFMHSFMIVFRVLCCEYIESMYDCMYVG ---DVSCIPFF 1020
\end{abstract}

Fig. 1 Pedigree of generalized epilepsy with febrile seizures plus (GEFS+) family with SCN1A mutation. a Identification of a SCN1A mutation c. $2830 \mathrm{~A}>\mathrm{C} / \mathrm{p} . \mathrm{N} 935 \mathrm{H}$ in the Chinese pedigree of family 1 . + wild type; $m$, mutant. b Sequencing data of exon 15 showed the c.A2803C/p.N935H. Arrow indicates nucleotide 2803, where a heterozygous a-c transversion resulted in an amino acid substitution p.N935H. The missense mutation was not found in 100 control alleles. $\mathbf{c}$ Evolutionary conservation of asparagine N935. Blue arrows indicate the positions of the missense mutations. Red shading indicates the transmembrane areas DIIS6. Amino acid sequence alignments of SCN1A and other alpha-subunit family members. (GenBank accession nos. P35498, P04774, Q99250, Q9NY46, P35499, Q14524, AAD15789.1, Q15858, Q9UI33, AAF24976.1, BAA07195.1, NP_956426.2, AAA79960.1, AAB59195.1) missense mutations showed that the mutation introduced residues with analogous properties (Fig. 1c).

\section{The GABRG2 mutation c.1287G > A/p.W390X}

In family 2 (Fig. 2a), proband III-4 is a 10-year-old boy. He developed the first attack of febrile seizures when he was 1 year and 3 months. The seizure type was GTCS, and the duration was 1-2 min. Subsequently, similar attacks occurred four to six times per year, and several attacks of AGTCS occurred. His psychomotor development was normal. At age 6 years, he was admitted to our hospital. Interictal video EEG and cranial computed tomography (CT) revealed no abnormality. The patient was administered valproate (VPA). The last febrile seizures occurred when he was 8 years and 5 months before our study. Since then, he has been seizure free. He was diagnosed with FS+. There were seven affected members (FS+ 6; FS 1) in the three-generation family. The affected members II-2, II-4, II-6, II-8, III-2, and III-4 had FS+. Member I-2 had a history of febrile seizures, but detailed data were unavailable.

The nonsense mutations c.1287G $>\mathrm{A} / \mathrm{p} . \mathrm{W} 390 \mathrm{X}$ in GABRG2 were identified in the family 2 (Fig. 2b). The nucleotide substitution c. $1287 \mathrm{G}>\mathrm{A}$ in exon 9 changed the

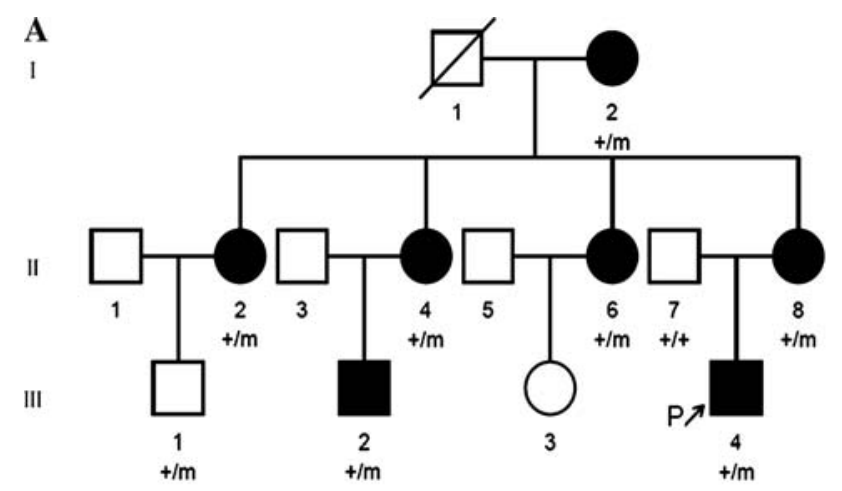

B

c. $1287 \mathrm{G}>\mathrm{A} / \mathrm{p} . \mathrm{W} 390 \mathrm{X}$

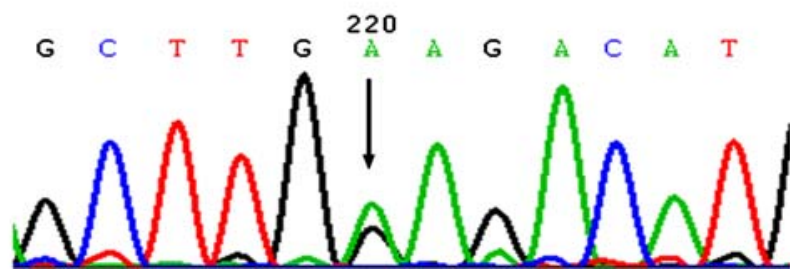

Fig. 2 a The c. $1287 \mathrm{G}>\mathrm{A} / \mathrm{p}$.W390X mutation in the GABRG2 gene cosegregates with generalized epilepsy with febrile seizures plus (GEFS+) in family 2. DNA was obtained from nine family members in three generations. The patient III-1 were mutation carriers but asymptomatic, illustrating the incomplete penetrance of the $G A B R G 2$ mutations. + wild type; $m$ mutant. b Electropherogram of the mutation in GABRG2 identified in family 2 . The nucleotide sequence of the relevant region of exon 9 of the proband is shown 
tryptophan codon TGG to nonsense codon TGA and truncated the channel protein in the intracellular loop between the third and fourth transmembrane segments. We sequenced all available family members and found it cosegregated with the GEFS+ phenotype. We found seven affected individuals (FS+ 6; FS 1) and one unaffected member carrying the mutations. We interpret the presence of the mutation in the asymptomatic individual III- 1 as an example of the incomplete penetrance of GEFS+. The penetrance rate of family 2 was about $87.5 \%$ (7/8).

\section{Discussion}

Phenotypes in GEFS+ families

GEFS + is inherited as an autosomal dominant trait with incomplete penetrance ranging from $62 \%$ to $89 \%$ in a small number of families (Scheffer and Berkovic 1997; Gerard et al. 2002; Bonanni et al. 2004). All 23 families were consistent with the characteristics of autosomal dominant inheritance; however, bilineal family history observed in three families $(3 / 23,13.0 \%)$ showed that two or more pathogenic genes may be involved in some GEFS + families.

GEFS + is a familial inherited epileptic syndrome characterized by phenotypic heterogeneity. In these 23 families, the affected members predominantly exhibited FS and FS+, which were major phenotypes of GEFS+. Rare phenotypes AGTCS, MAE, BECT, and partial seizures were also observed. No SMEI or idiopathic generalized epilepsy, childhood absence epilepsy and juvenile myoclonic epilepsy, was observed. These phenotypes shared the same mutations as other GEFS+ phenotypes in some GEFS+ families (Marini et al. 2003; Escayg et al. 2001).

\section{Mutation analysis}

\section{SCN1A mutation}

The SCN1A gene, encoding the voltage-gated sodium channel $\alpha-1$ subunit dominantly expressed in the central nervous system (CNS), is now the most clinically relevant gene for epilepsy. To date, approximately 170 SCN1A mutations have been identified in SMEI and borderline SMEI (SMEB), and about 16 missense mutations have been reported in GEFS + families (Harkin et al. 2007; Sugawara et al. 2001b; Mulley et al. 2005; Barela et al. 2006). To the best of our knowledge, mutation p.N935H in our study is novel, extending the distribution of SCN1A mutations to a population of Chinese origin. The SCN1A mutation rate was obviously higher in SMEI than in GEFS+: the mutation rate of SMEI was $33-100 \%$, whereas the rate in GEFS+ was 5-10\% (Mulley et al. 2005).
Consistent with previous studies, SCN1A mutation frequency $4.3 \%(1 / 23)$ in the GEFS + families is obviously lower than the detection rate in SMEI $(10 / 12,83.3 \%)$ in our study (unpublished data).

In addition, the types and locations of SCNIA mutations were associated with phenotypes. Most mutations in SMEI were de novo: two thirds were truncation, one third were missense mutations, and deletions of large exons were identified in some SMEI (Yamakawa 2006; Mulley et al. 2006). Almost all SCN1A mutations in GEFS+ were inherited missense mutations. The sodium channel alpha subunit folds into four domains (I-IV) of six transmembrane segments (S1-S6). In each domain, the voltage sensor is located in S4 segments and S5-S6 segments form the pore region. The SCN1A mutations associated with GEFS + were mainly distributed in segments outside S4S6. In the previously published 16 mutations of GEFS+ families, four mutations were on voltage sensor and three on the pore region (Pineda-Trujillo et al. 2005). Clinical correlations between phenotype and genotype have illustrated that SCN1A missense mutations on the important functional regions were more likely to be related to SMEI, which were considered to be the most severe phenotype of GEFS + in rare families (Nabbout et al. 2003; Ceulemans et al. 2004; Kanai et al. 2004). The mutation p.N935H (DIIS5-S6 linker) in our study was the fourth mutation located in the pore region in GEFS + families. It was cosegregated with the proband and his father in family 1 . However, no SMEI was observed in this family, although the mutation was located in the important functional regions.

Functional studies of the sodium channel $\alpha-1$ subunit with SCN1A mutations have demonstrated that either gain or loss of function of the mutations can result in GEFS+. The electrophysiological analysis on SCNIA mutations by patch clamp cannot fully explain molecular pathogenesis and phenotypes (Lossin et al. 2002; Ohmori et al. 2006). In some families, individuals with the same mutations had different phenotypes from asymptomatic carriers to FS to SMEI (Kimura et al. 2005; Mancardi et al. 2006). Genetic backgrounds and environmental factors may be associated with the incomplete penetrance and various severity of phenotypes.

\section{GABRG2 mutation}

The ligand-gated $\mathrm{GABA}_{\mathrm{A}}$ receptor is a heteropentameric chloride ion channel mediating rapid synaptic inhibition in the CNS generated by GABA. The $\mathrm{GABA}_{\mathrm{A}}$ receptor gene family includes several major classes of subunits with multiple subtypes, whereas the $(\alpha 1)_{2}(\beta 2)_{2} \gamma 2$ subunits are the most abundant combination in the brain. To the best of our knowledge, mutations in the GABRG2 gene encoding 
the $\gamma-2$ subunit of $\mathrm{GABA}_{\mathrm{A}}$ receptors were identified in five families with GEFS +, childhood absence epilepsy (CAE), and FS. Here we report a Chinese family with a novel GABRG2 mutation, bringing the total number of known mutations to six. This finding confirms the significant role of $G A B R G 2$ in GEFS+.

The truncation mutation p.W390X in our study may lead to the predictable terminations at amino acids 390. The location of the nonsense mutation p.W390X in family 2 was the second nonsense mutation in addition to p.Q351X of the GABRG2 gene. Both of them truncated the channel protein in the intracellular loop between the third and fourth transmembrane domains. These truncated proteins are likely to function as a dominant negative mutation, whereas the remaining normal genes show haploid insufficiency in the channel protein level (Harkin et al. 2002). The mutation cosegregated with the three-generation GEFS + family. Incomplete penetrance in the asymptomatic individual with the mutation illustrated that it was not a fully penetrant allele.

The first mutation of GABRG2 p.K289 $\mathrm{M}$ was found in a large French GEFS+ family comprising 17 affected members, most of whom had FS and some of whom developed epilepsies (Baulac et al. 2001). In an Australian family associated with p.R43Q mutation, the phenotypes predominantly exhibited FS and CAE (FS, 15; CAE, 8) (Wallace et al. 2001). Mutation screening of 135 German idiopathic absence epilepsies (IAEs) [CAE and juvenile absence epilepsy (JAE)] patients for $G A B R G_{2}$ was performed (Kananura et al. 2002). A splice donor site mutation (IVS6 $+2 \mathrm{~T} \rightarrow \mathrm{G}$ ) cosegregated a single nuclear family with CAE and FS. The low mutation rates of IAEs $(1 / 135,0.74 \%)$ indicated that $G A B R G 2$ is not a major gene in the pathogenesis of IAEs. Interestingly, the two siblings with CAE in the family with $G A B R G 2$ mutations had FS. Recently, missense mutation p.R139G was identified in a Belgian simple FS family (Audenaert et al. 2006). In a French GEFS + family associated with nonsense mutation p.Q351X, the proband had SMEI, but both his mother and brother had FS (Harkin et al. 2002). In family 2 of our study, there were seven affected members (FS+ 6; FS 1) with mutation p.W390X. Therefore, GABRG2 mutations were more likely to be the common pathogenic gene for FS and FS+; other modifier genes may contribute to other phenotypes.

\section{SCN1B mutation}

The sodium channel $\beta$-1 subunit gene, $S C N 1 B$, plays an important role for modulation of the inactivation of sodium channel alpha subunits. It was the first identified gene for GEFS+; however, to date, four mutations have been found to be associated with seven GEFS+ families (Scheffer et al. 2007). The reason for absence of $S C N 1 B$ mutation in our data was that $S C N 1 B$ mutations are less common than $S C N 1 A$ and GABRG2 mutations in GEFS+. Our limited data $(n=23)$ was another explanation.

In summary, this paper describes identification of two novel mutations in two unrelated GEFS+ families, extending the distribution of SCN1A and GABRG2 mutations to a population of Chinese origin. The positive findings of gene mutations reinforce the diagnosis of GEFS + as a member of Channelopathies at the molecular level. Although $S C N 1 A, S C N 1 B$, and GABRG2 genes are involved in the pathogenesis of GEFS+, they are rare pathogenic genes for GEFS+. The proportion of GEFS+ caused by the three genes was $8.7 \%(2 / 23)$. We failed to identify any mutations in the vast majority families, and other unidentified genes may be involved.

Acknowledgments We are very thankful to all members of the families who participated in our work. This research was supported by grants from Beijing Natural Science Foundation of China (No. 7072083).

\section{References}

Audenaert D, Schwartz E, Claeys KG, Depres L, Suls A, Dyck TV, Lagae L, Broeckhoven V, Macdonald RL, Jonghe PD (2006) A novel GABRG2 mutation associated with febrile seizures. Neurology 67:687-690

Barela AJ, Waddy SP, Lickfett JG, Hunter J, Anido A, Helmers SL (2006) An epilepsy mutation in the sodium channel SCN1A that decreases channel excitability. J Neurosci 26(10):2714-2723

Baulac S, Huberfeld G, Gourfinkel-An I, Mtropoulou G, Beranger A, Prudhomme JF, Baulac M, Brice A, Bruzzone R, LeGuern E (2001) First genetic evidence of GABA(A) receptor dysfunction in epilepsy: a mutation in the gamma2-subunitgene. Nat Genet 28:46-48

Bonanni P, Malcarne M, Moro F, Veggiotti P, Buti D, Ferrari AR, Parrini E, Mei D, Volzone A, Zara F, Heron SE, Bordo L, Marini C, Guerrini R (2004) Generalized epilepsy with febrile seizures plus (GEFS+): clinical spectrum in seven Italian families unrelated to SCN1A, SCN1B, and GABRG2 gene mutations. Epilepsia 45(2):149-158

Ceulemans B, Claes L, Lagae LG (2004) Clinical correlations of mutations in the SCN1A gene: from febrile seizures to severe myoclonic epilepsy in infancy. Pediatr Neurol 30:236-243

Commission on Classification and Terminology of the International League Against Epilepsy (1989) Proposal for revised classification of epilepsies, epileptic syndromes. Epilepsia 30:389399

Dibbens LM, Feng HJ, Richards MC, Harkin LA, Hodgson BL, Scott D, Jenkins M, Petrou S, Sutherland GR, Scheffer IE, Berkovic SF, Macdonald RL, Mulley JC (2004) GABRD encoding a proteinfor extra- or peri-synaptic GABA(A) receptors is a susceptibility locus for generalized epilepsies. Hum Mol Genet 13:1315-1319

Escayg A, MacDonald BT, Meisler MH, Baulac S, Huberfeld G, AnGourfinkel I, Brice A, Le Guern E, Moulard B, Chaigne D, Buresi C, Malafosse A (2000) Mutations of SCN1A, encoding a neuronal sodium channel, in two families with GEFS+2. Nat Genet 24:343-345 
Escayg A, Heils A, MacDonald BT, MacDonald BT, Haug K, Sander T, Meisler MT (2001) A novel SCN1A mutation associated with generalized epilepsy with febrile seizures plus and prevalence of variants in patients with epilepsy. Am J Hum Genet 68(4):866873

Gerard F, Pereira S, Robaglia-Schlupp A, Genton P, Szepetowski P (2002) Clinical and genetic analysis of a new multigenerational pedigree with GEFS + (generalized epilepsy with febrile seizures plus). Epilepsia 43:581-586

Harkin LA, Bowser DN, Dibbens LM, Singh R, Phillips F, Wallace RH, Richard MC, Williams DA, Mulley JC, Berkovic SF, Scheffer IE, Petrou S (2002) Truncation of the $\mathrm{GABA}_{\mathrm{A}}$ receptor $\gamma 2$-subunit in a family with generalized epilepsy with febrile seizures plus. Am J Hum Genet 70:530-536

Harkin LA, McMahon JM, Iona X, Dibbens L, Pelekanos JT, Zuberi SM, Sadleir LG, Andermann E, Gill D, Farrell K, Connolly M, Stanley T, Harbord M, Andermann F, Wang J, Batish SD, Jones JG, Seltzer WK, Gardner A, The Infantile Epileptic Encephalopathy Referral Consortium, Sutherland, Grant 1,2, Berkovic SF, Mulley JC, Scheffer IE (2007) The spectrum of SCN1Arelated infantile epileptic encephalopathies. Brain 130(3):843852

Kanai K, Hirose S, Oguni H, Fukuma G, Shirasaka Y, Miyajima T, Wada $\mathrm{K}$, Iwasa $\mathrm{H}$, Yasumoto $\mathrm{S}$, Matsuo $\mathrm{M}$, Ito $\mathrm{M}$, Mitdudome A, Kaneko S (2004) Effect of localization of missense mutations in SCN1A on epilepsy phenotype severity. Neurology 63:329334

Kananura C, Haug K, Sander T, Runge U, Gu W, Hallmann K, Rebstock J, Heils A, Steinlein OK (2002) A splice-site mutation in GABRG2 associated with childhood absence epilepsy and febrile convulsions. Arch Neurol 59:1137-1141

Kimura K, Sugawara T, Mazaki-Miyazaki E, Hoshino K, Nomura Y, Tateno A, Hachimori K, Yamakawa K, Segawa M (2005) A missense mutation in SCN1A in brothers with severe myoclonic epilepsy in infancy (SMEI) inherited from a father with febrile seizures. Brain Dev 27:424-430

Lossin C, Wang DW, Rhodes TH, Vanoye CG, George AL (2002) Molecular basis of an inherited epilepsy. Neuron 34:877-884

Mancardi MM, Striano P, Gennaro E, Madia F, Paravidino R, Scapolan S, Bernardina BD, Bertini E, Bianchi A, Capovilla G, Darra F, Elia M, Freri E, Gobbi G, Granata T, Guerrini R, Pantaleoni C, Parmeggiani A, Romeo A, Santucci M, Vecchi M, Veggiotti P, Vigevano F, Pistorio A, Gaggero R, Zara F (2006) Familial occurence of febrile seizures and epilepsy in severe myoclonic epilepsy of infancy (SMEI) patients with SCN1A mutations. Epilepsia 47:1629-1635

Marini C, Harkin LA, Wallace RH, Mulley JC, Scheffer IE, Berkovic SF (2003) Childhood absence epilepsy and febrile seizures: a family with a GABA(A) receptor mutation. Brain 126:230-240

Miller SA, Dykes DD, Polesky HF (1988) A simple salting out procedure for extracting DNA from human nucleated cells. Nucleic Acids Res 16(3):1215

Mulley J, Scheffer I, Petrou S, Dibbens LM, Berkovic SF, Harkin LA (2005) SCN1A mutations and epilepsy. Hum Mutat 25:535-542

Mulley J, Nelson P, Guerrero S, Dibbens L, Iona X, McMahon JM, Harkin L, Schouten J, Yu S, Berkovic SF, Scheffer IE (2006) A new molecular mechanism for severe myoclonic epilepsy of infancy: exonic deletions in SCN1A. Neurology 67:1094-1095

Nabbout R, Gennaro E, Bernardina BD, Dulac O, Madia F, Bertini E, Capovilla G, Chiron C, Cristofori G, Elia M, Fontana E, Gaggero R, Granata T, Guerrini R, Loi M, Selva LL, Lispi ML, Matricardi A, Romeo A, Tzolas F, Valseriati D, Veggiotti P, Vigevano F, Lallee L, Bricerelli FD, Bianchi A, Zara F (2003) Spectrum of SCN1A mutations in severe myoclonic epilepsy of infancy. Neurology 60:1961-1967

Ohmori I, Ouchida M, Ohtsuka Y, Oka E, Shimizu K (2002) Significant correlation of the SCN1A mutations and severe myoclonic epilepsy in infancy. Biochem Bioph Res Commun 295:17-23

Ohmori I, Kahlig KM, Rohdes TH, Wang DW, George AF (2006) Nonfunctional SCN1A is common in severe myoclonic epilepsy of infancy. Epilepsia 47(10):1636-1642

Pineda-Trujillo N, Carrizosa J, Cornejo W, Arias W, Franco C, Cabrera D, Bedoya G, Ruiz-Linares A (2005) A novel SCN1A mutation associated with severe GEFS + in a large South America pedigree. Seizure 14:123-128

Scheffer IE, Berkovic SF (1997) Generalized epilepsy with febrile seizures plus: a genetic disorder with heterogeneous clinical phenotypes. Brain 120:479-490

Scheffer IE, Harkin LA, Grinton BE, Dibbens LM, Turner SJ, Zielinski MA, Xu R, Jackson G, Adams J, Connellan M, Petrou S, Wellard RM, Briellmann RS, Wallace RH, Mulley JC, Berkovic SF (2007) Temporal lobe epilepsy and GEFS $^{+}$ phenotypes associated with SCN1B mutations. Brain 130:100 109

Singh R, Andermann E, Whitehouse WPA, Harvey AS, Keene DL, Seni M-H, Crossland KM, Andermann F, Berkovic SF, Scheffer IE (2001) Severe myoclonic epilepsy of infancy: extended spectrum of GEFS+? Epilepsia 42(7):837-844

Sugawara T, Tsurubuchi Y, Agarwala KL, Ito M, Fukuma G, MazakiMiyazaki E, Nagafuji H, Noda M, Imoto K, Wada K, Mitsudome A, Kaneko S, Monta M, Nagata K, Hirose S, Yamakawa K (2001a) A missense mutation of the $\mathrm{Na}^{+}$channel alpha II subunit gene $\mathrm{Na}_{\mathrm{v}} 1.2$ in a patient with febrile and afebrile seizures causes channel dysfunction. Proc Natl Acad Sci USA 98:6384-6389

Sugawara T, Mazaki-Miyazaki E, Ito M, Nagafuji H, Fukuma G, Mitsudome A, Wada K, Kaneko S, Hirose S, Yamakawa K (2001b) Nav1.1 mutations cause febrile seizures associated with afebrile partial seizures. Neurology 57:703-705

Wallace RH, Wang DW, Singh R, Scheffer IE, George AL, Phillips HA, Saar K, Johnson EW, Sutherland GR, Berkovic SF, Mulley JC (1998) Febrile seizures and generalized epilepsy associated with a mutation in the Na+-channel beta1 subunit gene SCN1B. Nat Genet 19:366-370

Wallace RH, Marini C, Petrou S, Harkin LA, Browser DN, Panchal RG, Williams DA, Sutherland GR, Mulley JC, Scheffer IE,

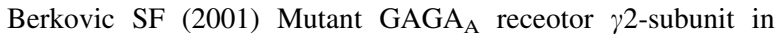
childhood absence epilepsy and febrile seizures. Nat Genet 28:49-52

Yamakawa K (2006) Na channel gene mutations in epilepsy-the functional consequences. Epilepsy Res 70S:S218-S222 